\title{
Cytomegalovirus associated neonatal pneumonia and Wilson-Mikity syndrome: a causal relationship?
}

\author{
F. Reiterer*, H.J. Dornbusch*, B. Urlesberger*, P. Reittner ${ }^{+}$, R. Fotter ${ }^{+}$, M. Zach**, H. Popper, \\ W. Müller*
}

Cytomegalovirus associated neonatal pneumonia and Wilson-Mikity syndrome: a causal relationship? F. Reiterer, H.J. Dornbusch, B. Urlesberger, P. Reittner, R. Fotter, M. Zach, H. Popper, W. Müller. (C) ERS Journals Ltd 1999.

ABSTRACT: Lung injury caused by intrauterine inflammation has recently been strongly implicated in the pathogenesis of Wilson-Mikity syndrome (WMS). This article supports this theory by suggesting a causative role of intrauterine cytomegalovirus (CMV) infection for the development of WMS.

A male premature infant, born at 33 weeks of gestational age, developed chronic lung disease compatible with WMS and diagnostic evaluation was positive for CMV infection.

High-resolution computed tomography scan and lung histology revealed typical features of WMS in association with signs of interstitial pneumonia. CMV was found in urine, breastmilk, bronchoalveolar lavage material and lung tissue from open lung biopsy. Follow-up after treatment with ganciclovir and steroids showed resolving lung disease at the age of 6,10 and 16 months, with lung function signs of mild obstruction.

Assuming that a chance coexistence of cytomegalovirus pneumonia and WilsonMikity syndrome is rather unlikely, it is possible that intrauterine cytomegalovirus infection caused a pattern of lung injury consistent with Wilson-Mikity syndrome. Further cases of Wilson-Mikity syndrome should be investigated as to a possible role of congenital infection.

Eur Respir J 1999; 13: 460-462.

Wilson-Mikity syndrome (WMS) is a chronic neonatal lung disorder of unknown aetiology occurring primarily in preterm neonates. It is characterized radiologically by alternating areas of cyst-like hyperaeration and infiltration developing within a few days or weeks after birth without preceding mechanical ventilation or high supplemental oxygen $[1,2]$.

The histological findings have been described as an immature alveolar pattern with areas of hyperinflation and collapse [3] and abnormal bombesin-containing neuroendocrine cells [4], with no signs of ventilator-induced injury of the airway epithelium as considered typical for bronchopulmonary dysplasia (BPD). Possible explanations for the pathogenesis include a disturbance in ventilation/perfusion secondary to immaturity of the lung [2], chronic hypoxia from hypoventilation and/or autonomic dysfunction [3] and response to congenital or perinatal infections [5]. The possible role of an intrauterine infection in WMS is suggested by high neonatal serum immonoglobulin (Ig)M, an association with chorioamnionitis and an increased level of leukocyte elastase in the tracheal aspirate at birth in patients who subsequently developed WMS [5]. However, a specific infectious agent has not yet been identified. This study reports a case of an infant in whom both cytomegalovirus (CMV) infection and signs of WMS were detected simultaneously, thus suggesting the possibility of a causal relationship.
Divisions of *Neonatology and **Pulmonology/Allergology, Dept of Pediatrics, Division of Radiology, Dept of Radiology, and \# Dept of Pathology, University of Graz, Austria.
Correspondence: F. Reiterer
Division of Neonatology
University Children's Hospital
8036 Graz
Auenbruggerplatz 30
Austria
Fax: 433163859678

Keywords: Cytomegalovirus pneumonia Wilson-Mikity syndrome

Received: February 21998

Accepted after revision August 81998

\section{Case report}

The patient, a 19 month old male, is the first child of a $36 \mathrm{yr}$ old female. The pregnancy was uncomplicated until the 25th week of gestation, when premature rupture of membranes caused oligohydramnion. The mother was treated with antibiotics for suspected chorioamnionitis and received five courses of betamethasone to accelerate lung maturation. At 33 weeks of gestation she spontaneously delivered a $1,860 \mathrm{~g}$ male. Immediately after birth the baby developed symptoms of respiratory distress with an oxygen demand of $30-50 \%$. Antibiotic therapy was started in the delivery room with cefuroxim and azclocillin. The chest radiograph was unremarkable. Although oxygen demand was decreased to room air within $2 \mathrm{~h}$, respiratory symptoms (tachypnoea and retractions) persisted. On day 4 the baby had an increased temperature and the radiograph displayed an area of consolidation in the right lung. Antibiotics were changed to imipenem and continuous airway pressure was applied via a mask. Respiratory symptoms improved temporarily.

At the age of 2 weeks the patient was transferred to the authors' department with the suspected diagnosis of perinatal pneumonia. He showed a mean respiratory frequency of 60 breath $\cdot \mathrm{min}^{-1}$ and carbon dioxide tension $\left(\mathrm{PCO}_{2}\right)$ of $6.9 \mathrm{kPa}(52 \mathrm{mmHg})$ and again required $30 \%$ oxygen to maintain the oxygen saturation above $90 \%$. Physical 


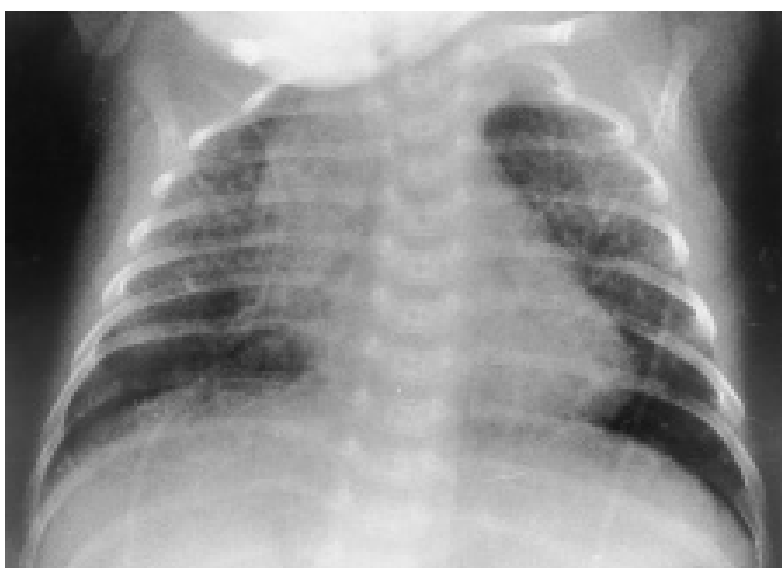

Fig. 1. - Chest radiograph on the 15th day of life showing a reticulonodular pattern with areas of focal hyperaeration throughout both lung and hyperaeration of the lower lobes.

examination of the lungs was normal. The chest radiograph (fig. 1) revealed a bilateral reticulonodular pattern with cyst-like hyperlucent areas, varying in size from $\sim 1-4 \mathrm{~mm}$ and hyperinflated lower lung fields. The alveolar-arterial oxygen difference increased to $7.3 \mathrm{kPa}(55 \mathrm{mmHg})$. Echocardiography showed signs of pulmonary hypertension. The antibiotic therapy was changed to erythromycin. Examination of the sputum for mycoplasma, chlamydia and Pneumocystis carinii produced negative results, as did the respective antibody titres. Virological screening, including CMV-IgM of the mother, the child and cord blood, was also negative. Since there was again no clinical improvement, erythromycin therapy was stopped after 2 weeks.

At the age of 1 month the search for a viral infection was intensified, and CMV-early antigen as well as polymerase chain reaction (PCR) for CMV deoxyribonucleic acid (DNA) in urine were found to be positive, followed by positive CMV-early antigen in sputum and positive PCR for CMV-DNA in breastmilk. IgM and pp65-immediateearly antigen remained negative. Based on these findings, the patient was started on ganciclovir $\left(10 \mathrm{mg} \cdot \mathrm{kg}^{-1} \cdot \mathrm{day}^{-1}\right)$, but no immediate radiological response was noted. For further diagnostic evaluation a high-resolution computed

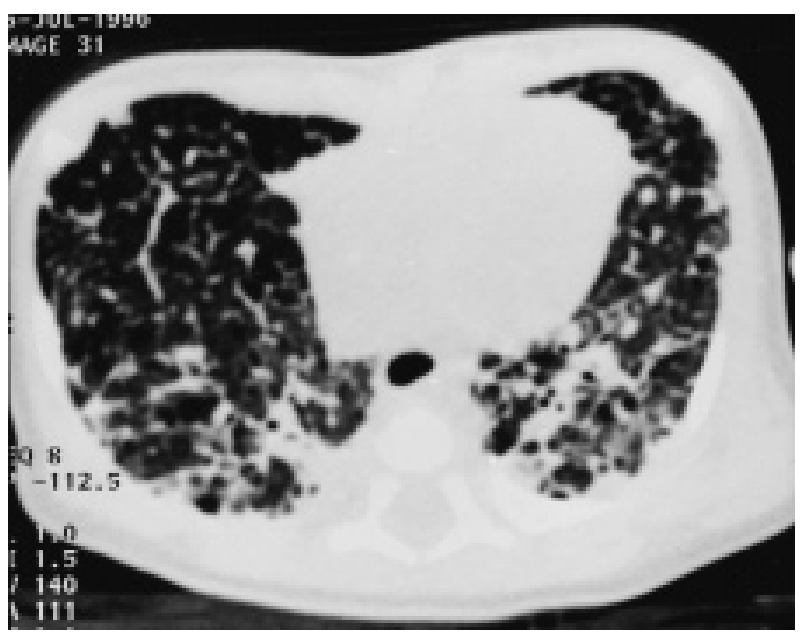

Fig. 2. - Inspiratory high-resolution computed tomography of lungs revealing hyperinflated cyst-like areas surrounded by thickened peribronchovascular interstitium and interlobular septa. tomography (HRCT) scan of the chest and bronchoalveolar lavage (BAL) were performed under general anaesthesia at 6 weeks of age.

HRCT images throughout both lungs showed; 1) a coarse thickening of the peribronchovascular and interlobular interstitium; 2) areas of hyperaeration with a lower lobe predominance; 3) parenchymal bands; and 4) distortion of vascular structures (fig. 2). Analysis of the BAL fluid showed nonspecific inflammation with lymphocytes and neutrophil granulocytes and the PCR for CMV-DNA was positive.

For histological differentiation between WMS, CMV pneumonia and other variants of interstitial lung diseases, a video-assisted thoracoscopic open lung biopsy was conducted 3 days later without complications.

Immunohistochemistry was performed using antibodies for smooth muscle actin (SMA), desmin, S100 protein, CD31 and 34, surfactant apoprotein A, neuron-specific enolase (NSE), chromogranin A (CGA), synaptophysin (SYNP) and bombesin/gastrin-releasing peptide (GRP).

In situ hybridization (ISH) was performed using probes for CMV, adenovirus-5 and respiratory syncytial virus (RSV). Histological evaluation of lung tissue on low-power and conventional stains showed dilated, hyperinflated lobules, interstitial inflammation and thickening. A constant finding was hyperplasia of smooth muscle cells in dilated alveoli (fig. 3), which could also be highlighted by immunohistochemical stains for SMA. In addition, there was an increase in neuroepithelial bodies in the peripheral lung and neuroendocrine cells in the bronchioles stained by both NSE and SYNP antibodies. Most of the cells were also stained by GRP antibodies. Another type of frequently found lung lesion was characterized by thickening of the alveolar walls, caused either by a lymphocytic and plasmocytic inflammatory infiltrate or by lung fibrosis. A few foci of organizing pneumonia were detected. By ISH for $\mathrm{CMV}$, positive signals were detected in endothelial cells of venules and capillaries, in a few type II pneumocytes and in alveolar macrophages. A reaction to surfactant apoprotein A stained only a few type II pneumocytes.

At 8 weeks of life, treatment with dexamethasone $(2$ $\mathrm{mg} \cdot \mathrm{kg}^{-1} \cdot \mathrm{day}^{-1}$ ) was started and the ganciclovir dosage was increased to $12 \mathrm{mg} \cdot \mathrm{kg}^{-1} \cdot \mathrm{day}^{-1}$. Within 2 weeks of dexamethasone therapy respiratory symptoms started to improve.

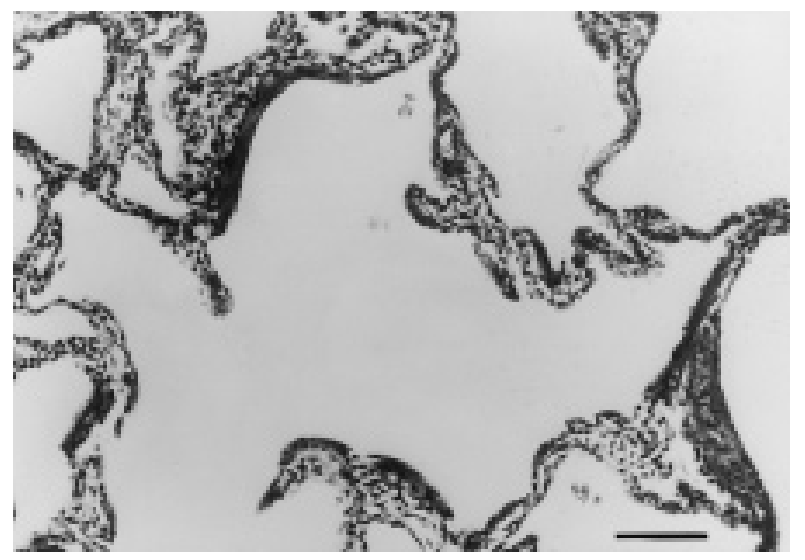

Fig. 3. - Open lung biopsy showing dilated alveoli and darker staining hyperplastic smooth muscle cells in the alveolar walls (Movat Pentachrome stain). (Internal scale bar $=200 \mu \mathrm{m}$.) 
Signs of bronchial obstruction became clinically evident and were treated by inhalation with salbutamol. Ganciclovir therapy was stopped after 6 weeks. Laboratory control at this time showed all parameters for CMV to be negative. The alveolar-arterial oxygen difference had decreased to $3.7 \mathrm{kPa}(28 \mathrm{mmHg})$ and echocardiography showed a significant improvement in pulmonary hypertension. At a corrected age of 2 months the patient was discharged from hospital. Oral corticosteroid therapy and salbutamol inhalations were continued. At this time he still had mild clinical symptoms of obstructive lung disease, but he was without additional oxygen demand. At the age of 6 months a control HRCT of the lungs was performed and showed significant improvement in the features described above. Follow-up investigation at the age of 10 and 16 months showed the patient to be in good clinical condition with lung function signs of mild obstruction.

\section{Discussion}

The case presented here suggests a relationship between an intrauterine CMV infection and WMS. Regarding the differential diagnosis of WMS and BPD, which is sometimes clinically difficult, several aspects make the diagnosis of WMS in this patient more likely. The patient had not been mechanically ventilated and received only very brief oxygen therapy. Although the effect of low levels of supplemental oxygen on the immature lung is not fully understood, this could have played only a relatively minor role in this patient. In addition, the histological findings in this case argued for WMS rather than BPD. Despite the similar radiographic appearance, the pathology of WMS is distinct from BPD [6]. Thus, the patient better fits into the clinical and pathological picture of WMS.

Infection and the tissue damage effected has been discussed as a factor in the pathogenesis of BPD [6]. By analogy, an infectious cause might also be discussed for WMS. FuJIMARA et al. [5] recently found high levels of polymorphonuclear leukocyte elastase- $\alpha_{1}$-proteinase inhibitor complex in the tracheal aspirate of neonates, decreasing thereafter, and speculated that the lung emphysema in WMS may be effected by proteolytic destruction of the lung parenchyma. These authors also found high serum IgM levels and chorioamnionitis. All of these findings strongly suggest an intrauterine infection in WMS. A specific infectious agent, however, has not yet been found. In the present patient the clinical, radiological and histological features were compatible with WMS and a spectrum of laboratory findings suggested CMV infection. An association between WMS and CMV infection could be interpreted in two ways. Firstly the coexistence of WMS and CMV infection could be a chance association. This possibility cannot be ruled out, but is unlikely, taking into account the rarity of the two conditions. Secondly, CMV infection may have played a causative role in the development of WMS. This hypothesis agrees with previous speculations about an infectious agent causing WMS. As symptoms of lung disease commenced at birth, however, interpretation of the presented case according to the latter hypothesis suggests congenital infection. This seems to contradict the observed negative serological findings at the age of 2 weeks. However, serological findings remained negative, even in the presence of CMV-positive urine, sputum, BAL and biopsy material. Thus, these negative serological findings suggest either a lack of an antibody response or faulty methodology, but they do not conclusively contradict the above hypothesis.

In summary, a patient was described with congenital cytomegalovirus infection mimicking Wilson-Mikity syndrome. Intrauterine cytomegalovirus infection may have resulted in retardation of lung development, inflammation and proteolytic tissue destruction. Further cases of Wilson-Mikity syndrome should be investigated regarding the role of congenital infections and particularly cytomegalovirus.

\section{References}

1. Wilson MG, Mikity VG. A new form of respiratory disease in premature infants. Am J Dis Child 1960; 99: 489499.

2. Swischuk LE. Wilson Mikity syndrome. In: Swischuk LE, ed. Imaging of the Newborn, Infant, and Young Child. Baltimore, MD, Williams-Wilkins, 1989; pp. 6872.

3. Hodgman JE, Mikity VG, Totter D, Cleland RS. Chronic respiratory distress in the premature infant. Wilson-Mikity syndrome. Pediatrics 1969; 44: 179-195.

4. Gillan JE, Cutz E. Abnormal pulmonary bombesin immunoreactive cells in Wilson-Mikity syndrome (pulmonary dysmaturity) and bronchopulmonary dysplasia. Pediatr Pathol 1993; 13: 165-180.

5. Fujimura M, Kitajima H, Nakayama M. Increased leukocyte elastase of the tracheal aspirate at birth and neonatal pulmonary emphysema. Pediatrics 1993; 92: 546-569.

6. Northway WH. An introduction to bronchopulmonary dysplasia. Clin Perinatol 1992; 19: 489-495. 